\title{
Implementation of Dual Use Fiber Optic Communications Laboratories
}

\author{
doi:10.3991/ijoe.v7i2.1637 \\ Akram Abu-aisheh ${ }^{1}$, Sameer Khader ${ }^{2}$, and Omar Hasan ${ }^{3}$ \\ ${ }^{1}$ University of Hartford, West Harford, CT, USA \\ ${ }^{2}$ Palestine Polytechnic University, Hebron, Palestine \\ ${ }^{3}$ Princess Sumaya University for Technology, Amman, Jordan
}

\begin{abstract}
Given the wide use of fiber optic technology in industry and high-end consumer electronics, the use of optical fiber should be more widely taught; however, limited available resources in the provision of laboratory hardware has increasingly marginalized the educational quality of fiber optic communications courses. This paper presents two models for implementing fiber optic communications laboratories for both on-site and distance learning environments. The dual use of these set-ups allows for a highly cost effective and pedagogically rigorous mechanism of instruction for students.
\end{abstract}

Index Terms-Remote, E-Learning, Fiber Optic, FPGA.

\section{INTRODUCTION}

The availability of low-cost plastic fiber, detectors, and data communications grade light emitting diodes (LEDs) makes suitable educational materials within financial reach; however, there is a need for the development of laboratory set-ups based on the use of these components. The needed set-up should be suitable to demonstrate the principles of fiber optic communications.

This paper presents two fiber optic communications platforms in which experiments can be implemented either in the lab or in a distance learning environments. In the first set-up [1], we present a dual use low cost FPGAbased fiber optic communications toolkit that students may use in the lab or take home to perform experiments in a distance learning environment. In the second set-up [2], we present a dual use computer-controlled optical switch matrix with the Emona FOTEx trainer and $\mathrm{Na}-$ tional Instrument's ELVIS II platform. Students may use this set up either in the lab or remotely. Both set-ups have been used in a graduate fiber optic communication course and undergraduate fiber optic technology course.

The technological viability of distance laboratories and their effectiveness in delivering a worthwhile laboratory experience in teaching communication courses have been validated by a growing body of work by many researchers around the world. Limitations on equipment access and funding reduce the availability of laboratory resources in many institutions. Remote labs help alleviate this problem by increasing access and simultaneously reducing cost [3]. The architectures and designs of remote labs have improved in the past five years [4-7].

\section{REMOTE LABORATORIES \& DistANCE LEARNING}

A remote laboratory is defined as a computercontrolled laboratory that can be accessed and controlled externally over some communication medium. Remote laboratories allow users to perform experiments and laboratory tasks over the Internet without being near the actual equipment. In the simplest case, the remote laboratory server can be an experiment connected to a computer through a standard interface and with the host computer connected to the Internet.

Distance learning, also referred to as e-learning, can be used to help universities and technical colleges overcome the limitation of scarce resources in the provision of laboratory hardware and infrastructure. One approach that can be implemented to overcome this problem is to expand elearning activities in programs with limited resources to take advantage of online computer-based technology.

The objective of using dual use platforms is not to replace the onsite laboratory experience, but rather to supplement it with a system that provides continuous access to the same experiments remotely via the Internet or in a hands-on format as presented in the case of the FPGA set-up. The educational optical fiber data communications platform can be used in a hands-on fashion either in the lab or outside the lab since only a PC is needed to be used with the toolkit. The FOTEX-ELVIS II platform can be used in hands on environment in the lab or remotely via a computer connected to the internet.

The next two sections in this paper present two dual use platforms. For each teaching environment, sample experiments were presented to clarify the differences between these two teaching environments. Both set-ups were implemented in an undergraduate fiber optic technology class and in a graduate fiber optic communications class, and each set-up has its own merits to be considered for fiber optic laboratory platforms.

\section{EDUCATIONAL OPTICAL FiBER COMMUNICATIONS TOOLKIT PLATFORM}

The main motive for developing and implementing the use of the fiber optic communications educational toolkit was the need for a low cost laboratory alternative at the University of Hartford to supplement two courses offered in this field. This FOC educational toolkit provides both undergraduate and graduate engineering students with a new way to study the physical layer of fiber optic communications systems

The fiber optic communications educational toolkit platform presents a toolkit that students can use to investigate the physical layer in an optical fiber based network. The required test gear includes a multimeter and an oscilloscope. Given the use of photo diodes, an optical power 
meter is entirely optional. At the core of the toolkit is the optical fiber toolkit adapter card which provides the transmitter and receiver electronics. The adapter board attaches to an off-the-shelf field programmable gate array (FPGA) development board, which provides the digital aspect of the data link.

The choice of using an FPGA provides a level of flexibility, allowing the toolkit to be used in other curriculum as well. Further, with a soft-core processor, the toolkit can be used to implement a modest microprocessor system. The fiber optic toolkit is useful in undergraduate classroom or laboratory settings, as well as in independent projects. This toolkit was used in two fiber optic communications classes at the University of Hartford.

The fiber optic toolkit provides a low-cost means for students to examine a practical data link. Such a data link can be constrained in at least two ways. Performance can be limited by the optical power budget or by the available bandwidth. The power budget is basically the difference between the transmitter coupled power and receiver sensitivity. Bandwidth is related to pulse spreading which involves inter-symbol interference. Given the significant loss and modest bandwidth, plastic fiber is useful for students to investigate such design constraints.

In particular, the kit uses Eska brand one millimeter core step-index plastic fiber, manufactured by Mitsubishi [8] The LED and photo diodes use simple connectorless type optics. The optical fiber is easily cut to length with a sharp blade. Given our use of photo diodes in the toolkit, an optical power meter is entirely optional.

The definition of decibel units is well known to be the ratio of two relative power values as given in (1). The advantage in using decibels is that equations become simpler. Given the squared relationship of the power dissipated by a resistor, with respect to voltage, (2) is true for resistors.

$$
\begin{aligned}
& P_{\mathrm{dB}}=10 \log \left(\frac{P}{\mathrm{P}_{\text {Ref. }}}\right) \\
& P_{\text {RES-dB }}=20 \log \left(\frac{V_{1}}{V_{\text {Ref. }}}\right)
\end{aligned}
$$

The programmable logic device (PLD) provides students new opportunities to study data communications. PLDs range from simple logic array devices used for glue logic and the complex programmable logic device (CPLD) that replaces many small chips, to the high density field programmable gate arrays (FPGA) capable of implementing an entire system on just one chip. For the sake of convenience we selected the Spartan-3 Starter board [9] in Figure 1 which provides a Spartan-3 FPGA device. The software tools that we use to configure the FPGA are free or are available at little cost.

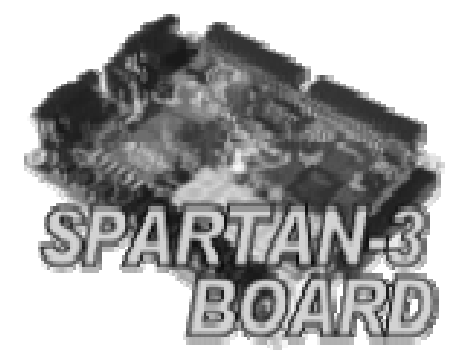

Figure 1. Spartan-3 FPGA development board
The development board has simple input and output devices, a clock generator, and other features. But in particular the board has expansion connectors for adapter cards. We use a custom adapter card to provide the transmitter and receiver electronics.

\section{SIMPLE EXPERIMENT: USING THE EDUCATIONAL OPTICAL FIBER DATA COMMUNICATIONS TOOLKIT}

Several Experiments were developed and implemented using the educational FOC toolkit. Experiments include:

1. Fiber Optic Link Linearity.

2. FOC Link Attenuation

3. FOC Link Dispersion

4. Data transmission

Power budget calculation is a useful tool for considering how optical power can be a constraining factor in fiber optic links design. This fiber optic communications toolkit can be used for Attenuation Experiments. The difference between the transmitter power and the minimum required power at the receiver is the amount of power available to the link, which comprises the sum of all the losses and margin. As given in (3).

$$
P_{\mathrm{tx}}-P_{\mathrm{rx}}=\sum P_{\mathrm{loss}}+P_{\mathrm{m}}
$$

In most cases in this paper, in comparing power values we assume decibels, as in (1). With optical fiber, it is sometimes useful to express power in $\mathrm{dBm}$ units, where the reference is one milli Watt. In those cases where we consider an actual power level, we use $\mathrm{dBm}$ units.

The circuit in Ficure 2 is the basic circuit for studying linearity and fiber optic cable attenuation. The LED load resistor is selected to adjust the LED current and the photo diode load resistor sets the sensitivity of the receiver. Given that the LED forward voltage is nearly constant, we expect to see a linear relationship between the transmitter optical output and the receiver currents.

Measurements were taken with an ordinary voltmeter using the set-up given in Figure 3. An example response of the photo diode load resistor voltage with respect to the LED current is given in Figure 4. The LED is an IFE97 [10] with 660nm wavelength and the photo diode is an IFD91 [11] device. The fiber used for Figure4 is one meter long. The fitted line did not consider the first three data points.

This shows that for moderate current, the coupled power is approximately linear with respect to the LED current. Likewise the photo diode current and load resistor voltage are also approximately linear with respect the received optical power.

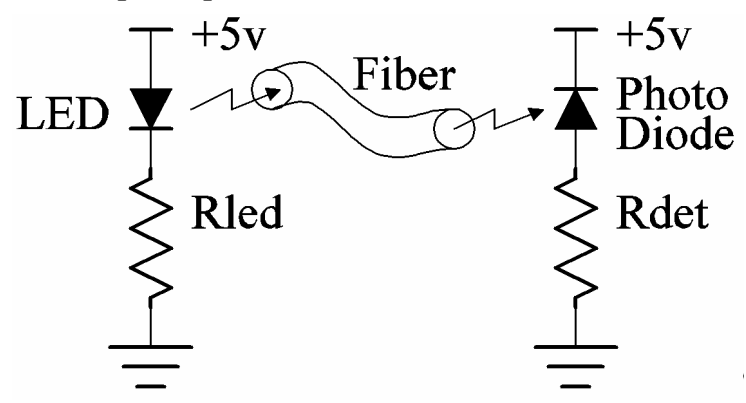

Figure 2. Linearity and attenuation test 
PAPER

IMPLEMENTATION OF DUAL USE FIBER OPTIC COMMUNICATIONS LABORATORIES
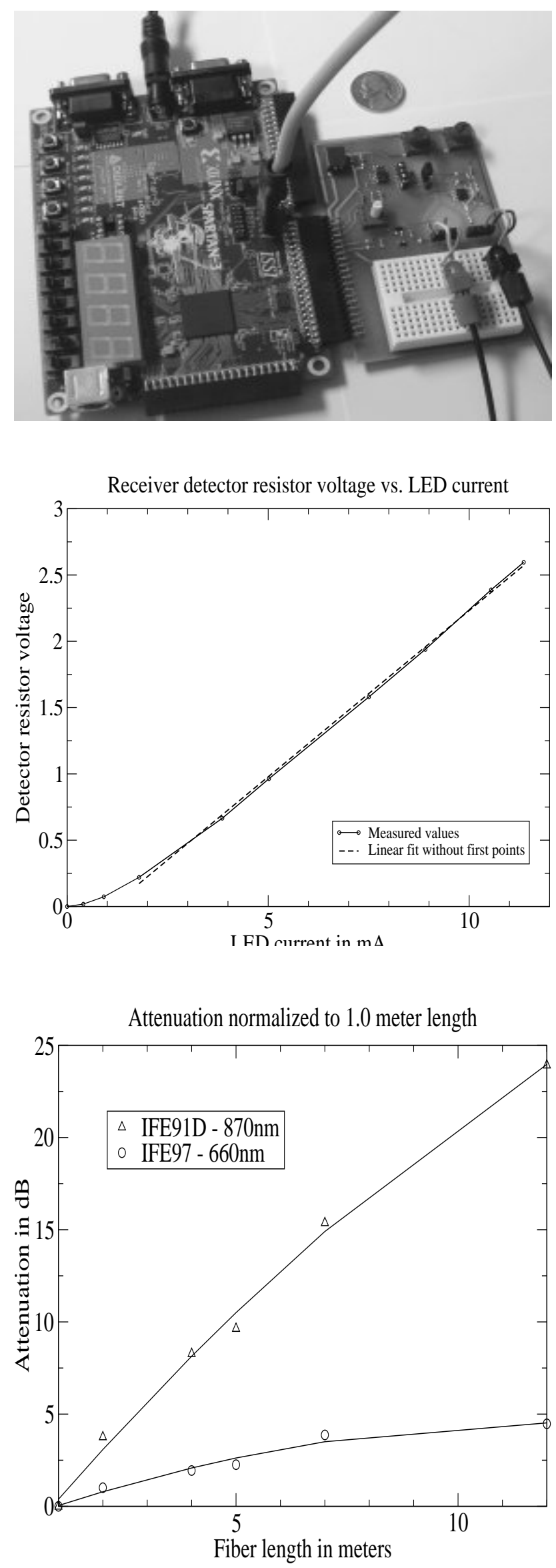

Figure 5. Attenuation versus fiber length
The circuit in Figure 2 can be used to find the optical fiber attenuation with respect to fiber length. An IFE91D [12] device with $870 \mathrm{~nm}$ wavelength is used to consider attenuation dependence on wavelength. The loss in a fiber can be predicted with the linear approximation of decibels over a standard distance.

To examine this approximation, students use optical fiber cut to various lengths to examine how end to end attenuation is related to the fiber length. In figure 6 , the attenuation in a one meter link is used as the reference, so that lengths longer than one meter have positive attenuation.

There are two principal observations for students to consider. The first one is that attenuation is dependent on wavelength and second that attenuation is different for shorter than for longer lengths of fiber. The dependence on wavelength is a property of the plastic used. One reason for the second observation is that in coupling light to a fiber there are unstable modes that dissipate in the first few meters. Once the unstable modes dissipate, the attenuation becomes nearly constant.

In our own measurements, for $660 \mathrm{~nm}$ wavelength the attenuation is approximately $0.75 \mathrm{~dB} / \mathrm{m}$ for $1 \mathrm{~m}$ length and $0.20 \mathrm{~dB} / \mathrm{m}$ for $10 \mathrm{~m}$ length. The data sheet for $\mathrm{GH} 4001$ Eska brand fiber[7] specifies a comparable worst case attenuation of $0.17 \mathrm{~dB} / \mathrm{m}$ at $650 \mathrm{~nm}$ for kilometer lengths of fiber. For $870 \mathrm{~nm}$ wavelength, attenuation was found to be approximately $2.7 \mathrm{~dB} / \mathrm{m}$ for $1 \mathrm{~m}$ lengths and $1.8 \mathrm{~dB}$ for $10 \mathrm{~m}$ length.

\section{Using the Educational Optical Fiber Data COMMUNICATIONS TOOLKIT TO TEST SYSTEM BANDWIDTH}

The overall response time and corresponding bandwidth causes pulse spreading which can degrade performance. The overall response time $T_{o}$ for a simple LED driven optical fiber data link is composed of its three components' response times below as given in (4). The fiber response time in particular is approximately proportional to the fiber length $L$. Note that because of the rootsquare relation for any one term to dominate its value must be significantly larger than the others.

$$
T_{o}=\sqrt{T_{\mathrm{tx}}^{2}+T_{\mathrm{f}}^{2}+T_{\mathrm{rx}}^{2}}
$$

- $T_{o}=$ Overall response time

- $T_{\mathrm{tx}}=$ Transmitter response time

- $T_{\mathrm{f}}=L \cdot \Delta t=$ Fiber spreading

- $T_{\mathrm{rx}}=$ Receiver response time

The first order low-pass filter model is particularly helpful. Here we consider a low-pass filter with time constant $\tau$ and D.C. gain $K$. The filter cutoff frequency is $F_{c}$ and the step response is $h(t)$.

A relationship between rise time and bandwidth is particularly useful. In considering the time to rise from $10 \%$ to $90 \%$ of the final value, we solve (5) and (6) to produce the familiar approximation in (7). 
PAPER

$$
\begin{aligned}
& H(s)=\frac{K}{1+\tau s} ; \text { where } \\
& h(t)=K\left(1-e^{-t / \tau}\right) \\
& t_{r}=\frac{-\ln (0.1)+\ln (0.9)}{2 \pi F_{c}} \approx \frac{0.35}{F_{c}} \mathrm{sec}
\end{aligned}
$$

We next consider the actual fiber. Optical dispersion is the spreading that occurs to a light pulse as it travels along an optical fiber, as in Figure 6. Here we consider dispersion due to the various optical modes that appear in a plastic fiber. The idea is that a light ray traveling straight down a fiber follows a shorter path and will arrive at the receiver sooner than a ray that reflects along inside the fiber. As outlined by Weinert, (8) is the measured spreading per unit length caused by the fiber and (9) predicts the spreading per unit length based on characteristics of the fiber, where NA is the numeric aperture, $c_{0}$ is the speed of light in a vacuum, and $n_{1}$ is the refractive index of the fiber core.

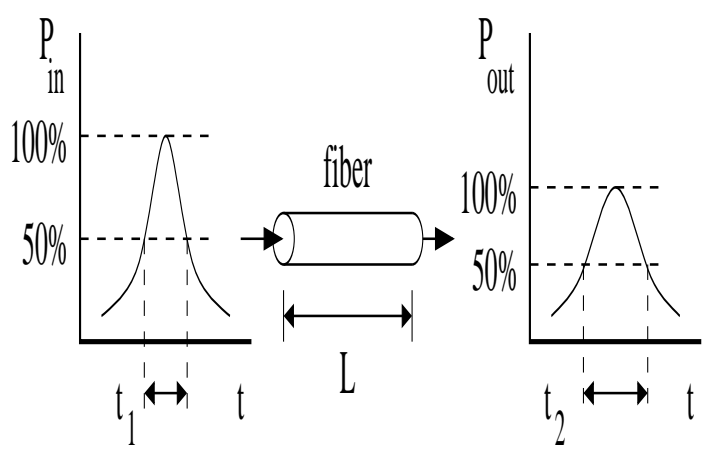

Figure 6. Pulse dispersion in optical fiber

$$
\begin{aligned}
& \Delta t=\frac{\sqrt{t_{2}{ }^{2}-t_{1}{ }^{2}}}{L} \\
& \Delta t=\frac{\mathrm{NA}^{2}}{2 c_{0} n_{1}}
\end{aligned}
$$

In using the values from the optical fiber data sheet[7] (NA $=0.5, n=1.49$ ), we estimate that the spreading is approximately $0.2796 \mathrm{~ns} / \mathrm{m}$, which is relatively small. Given the attenuation, it is safe to say that most plastic fiber optic data links are less than $100 \mathrm{~m}$ in length.

It is more likely that a plastic fiber optic data link driven by an LED will be limited by attenuation or the electrical components themselves, rather than the bandwidth of the optical fiber. Consider that for a $100 \mathrm{Mbs}$ data link the bit period of 10ns is approximately 35 times larger than the modal dispersion in one meter of this particular fiber.

\section{EMONA's FOTEX COMMUNICATIONS TRAINER NI'S ELVIS II EDUCATIONAL PLATFORM}

The FOTEx trainer is a plug-in module for the ELVIS platform. The FOTEx- ELVIS II bundle enables us to use hands-on approach in teaching fiber optic communica-

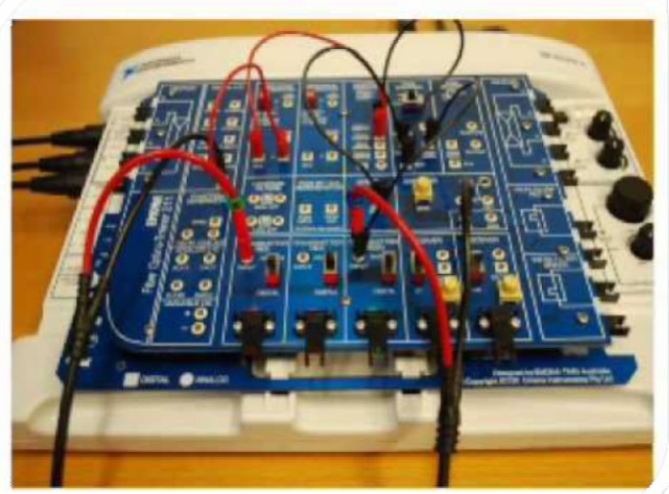

Figure 7. FOTEx-ELVIS II Set-up

tions concepts remotely using LabVIEW. We will show that by using the FOTEx-ELVIS II-based remote laboratory, as shown in Figure 7, students can log in and perform fiber optic experiments, set up a bandwidth limited signal and explore its spectral composition, and much more.

The online instrumentation can be evaluated by first having some basic knowledge about the NI ELVIS equipment. The bulk of measurements needed to test and repair electronics systems can be performed with just these devices. Similarly, the DC power supply and function generator are used in wide range to power the equipment and to provide a variety of AC signals. All these essential hardware devices/blocks in the laboratory are now in a single unit called the DATEx, which possesses the capabilities needed for implementing dozens of telecommunications experiments.

Fiber optic communications remote experiments make use of the FOTEx telecommunications trainer kit together with the NI ELVIS platform, and LabVIEW running on a PC. Thus, for e-learning, it is sufficient to take advantage of this infrastructure by simply logging on to a computer equipped with suitable interface circuits.

Fiber Optic communications experiments related to this setup have been prepared for students with only basic knowledge of mathematics and limited background in physics and electricity. The experiments that can be performed using the proposed setup are ideal for implementation in a remote environment.

\section{USING THE FoteX - ElVIS II SET-UP TO DEMONSTARTE WAVELENGTH DiVISION MULTIPLEXING}

Wavelength Division Multiplexing (WDM) is a technology used in fiber optic communications to combine optical signals. WDM uses a single fiber to transmit multiple optical signals. It does this by breaking up the signal into different wavelengths. The various wavelengths indicate that different amounts of data are being transmitted simultaneously. This results in an increase in capacity and allows bidirectional communication over any single fiber. For WDM to work properly there needs to be a multiplexer and a demultiplexer on the fiber. A multiplexer at the transmitter takes the signals and joins them together. Once the signals reach the demultiplexer, the joined signals are split apart at the receiver as given in figure 8 . 


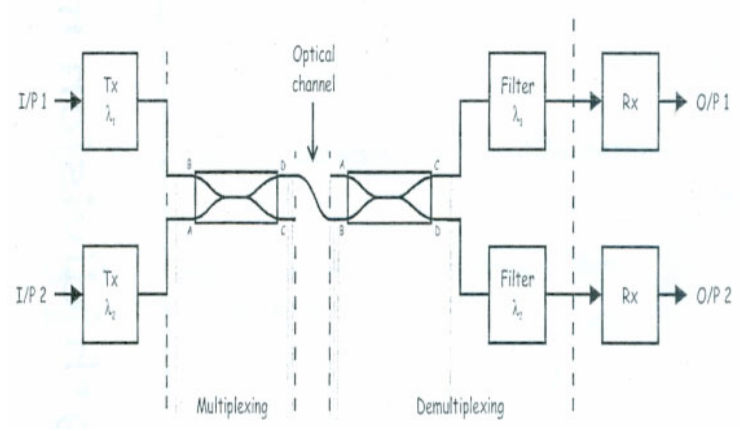

Figure 8. WDM Experiment block diagram

In this experiment, the FOTEx coupler module is used for combining optical signals. The Red Transmitter module was selected and its Mode Control was set to analog. Then, the Mode control of the other Red Transmitter module was turned to Digital. The Gain Range control of both Receiver modules were turned to L0 and the Variable Gain control of both Receiver modules were fully clockwise.

In like manner, students learn how to transmit two discrete message signals simultaneously along an optical fiber in the same direction using wavelength division multiplexing. The steps students need to follow in order to perform this experiment are the following:

- Ensure that the NI Elvis II power switch at the back of the unit is off

- Carefully plug the Emona FOTEX experimental add in module into ELVIS II

- Insert the holding screws to secure the FOTEX module to the NI ELVIS II

- Connect the ELVIS II to the pc using the USB

- Turn on the NI ELVIS II power switch at the rear to the unit

- Turn on the proto typing Board power switch at the top right corner near the power indicator

- Turn on the PC and let it boot

- Select one of the red LED Transmitter modules and set its mode control to analog

- Select one to the receiver modules and sets its gain control to HI

- Turn the same Receiver module Variable Gain control fully clock wise.

- Connect the set up in figure 9. The output on the oscilloscope is given in figure 10 .

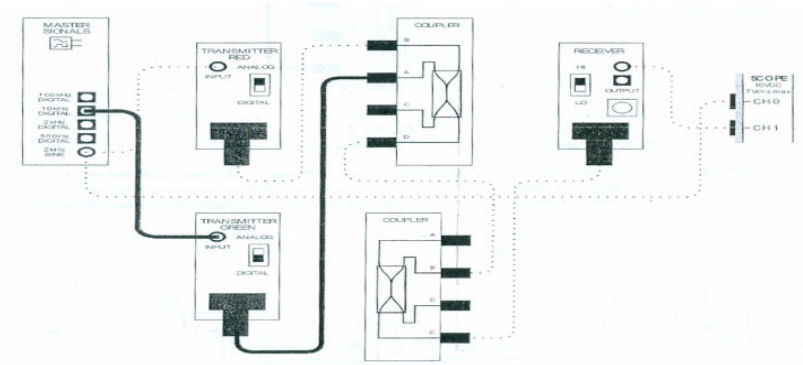

Figure 9. Experimental set up for the transmission of two signals over one channel -an analog message and a digital message

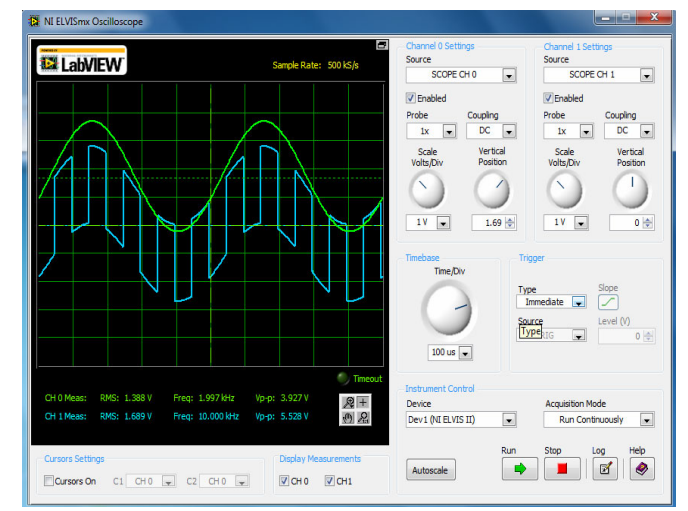

Figure 10. Experimental Output signals for the transmission of two signals over one channel -an analog message and a digital message

\section{STUDENTS EXPERIENCE USING THE FOTEX TRAINER IN A HANDS ON ENVIROMENT}

Two surveys were conducted to get students feedback about the use of the FOTEx and DATEx communication trainers' set-ups in the lab. The survey results for using the DATEx trainer set-up are given in [1]. The following questions about students experience in using the FOTExELVIS II set up in a fiber Optic Communications course were used for the survey:

1. Do you feel comfortable using the FOTEx-ELVIS setup?

2. Where the FOTEx -ELVIS setup experiments clearly written?

3. Where the FOTEx -ELVIS setup experiments procedures easy to follow?

4. Did the use of the FOTEx -ELVIS setup increase your interest in the labs?

5. Did you have difficulties in configuring the FOTEx ELVIS setup software?

6. Did you have difficulties in configuring the FOTEx ELVIS setup hardware?

7. Did you experience difficulties in performing any Fiber Optic Communications experiment?

8. Was the FOTEx -ELVIS lab setup very valuable in terms of learning?

9. Overall, do you support the use of the FOTEx ELVIS setup in this course?

The survey included 15 students enrolled in a graduate fiber optic communications engineering course, and the results of the survey are illustrated in Figure 11 where the blue (light) bar represents a "YES" answer and the red (dark) bar represent a "NO" answer. The X-axis represents the question number, and the $\mathrm{Y}$-axis represents the frequency of each answer. As given in the graph, there is a total agreement among all students that they feel comfortable using the FOTEx-ELVIS setup an that FOTEx ELVIS setup experiments clearly written.

The results given in figure 11 shows that there is an agreement among all students that they feel comfortable using the FOTEx-ELVIS setup an that FOTEx -ELVIS setup experiments clearly written. Also, all students surveyed support the use of the FOTEx -ELVIS setup. 
PAPER

IMPLEMENTATION OF DUAL USE FIBER OPTIC COMMUNICATIONS LABORATORIES

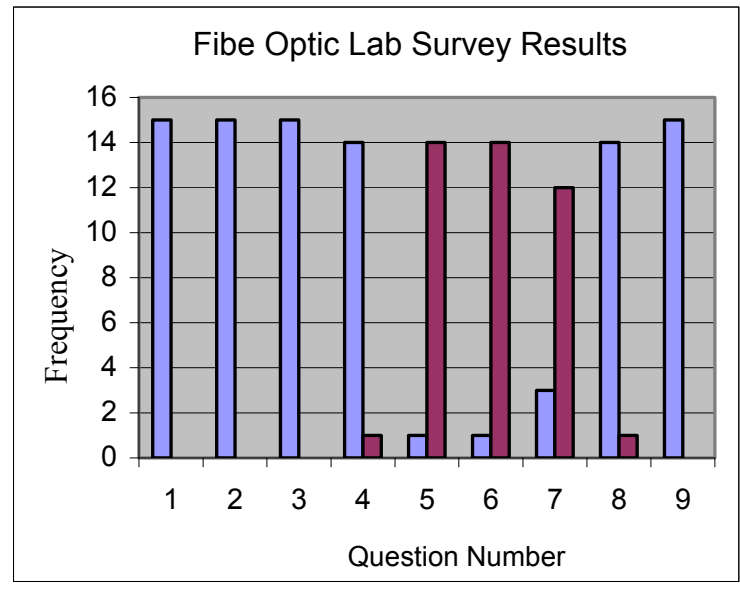

Figure 11. ELVIS II-FOTEX Fiber Optic Communications Survey Results

\section{CONCLUSION}

In this work, we presented two setups that can be used to perform advanced fiber optic communications experiments on site or remotely in a distance learning environment. Comparing the two set ups after using them in both engineering and engineering technology classes, the authors conclude that the FOTEx communication trainer with NI ELVIS II platform is more appropriate for timelimited laboratory experiments where emphasis is on process, while the educational optical fiber data communications toolkit platform is more appropriate for advanced electrical engineering course projects where emphasis is on design and there is more time available to complete the work.

The main advantage of the use of the FOTEx-ELVIS II platform is the ease of use while the main advantages that the FOC ETK has over the FOTEx-ELVIS II platform include lower cost, flexibility of experiment design, and the flexibility in use for design projects.

The results of the survey conducted about the use of the ELVIS II-FOTEX fiber optic communications set-up in the lab were very positive, and it shows that the FOTEx-ELVIS II set-up is a user friendly that has a fast learning curve. The next step is to implement the use of this set up with an optical switch matrix and use the setup in a collaborative distance learning environment.

The Fiber Optic Communications educational toolkit platform provides undergraduate and graduate engineering students with a low cost and flexible tool to study high speed fiber optic communication systems. As an introductory tool, the FOC ETK allows for a deductive approach, to investigate existing systems. Advanced students ready for an inductive approach can use the toolkit in their projects. In addition to the flexibility afforded by an FPGA, the accompanying development board should be particularly flexible in the discretion afforded to the instructor.

\section{REFERENCES}

[1] Hill, J. and Abu-aisheh, A., "Educational Optical Fiber Data Communications Toolkit," ASEE Conference Proceedings, Pittsburg, PA. 2008
[2] Abu-aisheh, A., Eppes, T, and Al-Zoubi, Abdullah, "Implementations of a Virtual RF and Digital Communications Laboratory for E-Learning". The International Journal of on-line Engineering (IJOE). Spring 2010.

[3] Eppes, T. and Schuyler, P., "A Robust and Scalable Distance Laboratory Platform" Proceedings of the 2004 Annual Conference \& Exposition, Session 2426.

[4] Cooney, E. \& Shriver, A. "Remote Control of a Robot Using LabVIEW and the World Wide Web", Proceedings of the 2001 ASEE Conference \& Exposition, Session 2526.

[5] Plummer, Mitty et al. "A Circuits II Laboratory Accessible by Internet", Proceedings of the 2002 ASEE Annual Conference, Montreal, Quebec Canada, June 2002.

[6] Malki, Heidar et al. "Web-based Control Systems Laboratories", Proceedings of the 2003 ASEE Annual Conference, Nashville, Tennessee, June 2003.

[7] Kolla, Sri et al. "Internet-Based ON/OFF Controller Using LabVIEW", Proceedings of the 2003 ASEE Annual Conference, Nashville, Tennessee, June 2003.

[8] Mitsubishi International Corp, http://www.mitsubishicorpus.com/ourbusiness_productsandservices.do? $\mathrm{id}=29$

[9] Digilent, Inc., http://www.digilentinc.com/, April 2, 2006

[10] Industrial Fiber Optics IFE97 660nm LED, http://ifiberoptics.com/leds/IFE97.pdf, April 22, 2006

[11] Industrial Fiber Optics IFD91 photo diode, http://ifiberoptics.com/leds/IFD91.pdf April 22, 2006

[12] Industrial Fiber Optics IFE91D 870nm LED, http://ifiberoptics.com/leds/IFE91D.pdf, April 22, 2006

\section{AUTHORS}

AKRAM ABU-AISHEH is an Associate Professor of Electrical and Computer Engineering at the University of Hartford where he has served as the assistant chair of the Electrical and Computer Engineering Department and director of the electronic and computer engineering technology program for two years. Dr. Abu-aisheh has a doctorate in Optical Communications from the Florida Institute of Technology and Master of Science and Bachelor of Science degrees in Electrical Engineering from the University of Florida. Dr. Abu-aisheh may be contacted at abuaisheh@hartford.edu.

SAMEER KHADER is an Associate Professor of Electrical and Computer Engineering at Palestine Polytechnic University (PPU) - Palestine. He is a director of Power Electronics \& Signal Processing Research Unit at PPU. Before that he served for ten years as university academic provost, dean of College Engineering \& Technology at PPU. His research interests include Electrical machines, Power Electronics Converters, Renewable Energy Sources and Smart Grids, in addition to engineering education. Dr. Khader may be contacted at sameer@ppu.edu

OMAR HASAN received his BS degree from New Mexico state university 1987, and received his MSC and $\mathrm{PhD}$ in communications engineering from New Mexico state university 1991 and 1996 . He served as department head for communications engineering at Princess Sumaya university 2005-2010 and currently he is the dean of student affairs at Princess Sumaya university. Dr. Hasan can be contact ohasan@psut.edu.jo.

This article is an extended version of a paper presented at the IEEE EDUCON2011 Conference, held from April 4th-6th, 2011, in Amman, Jordan. Received April 16 ${ }^{\text {th }}, 2011$. Published as resubmitted by the authors April 29, 2011. 